may carry a greater risk of long-term sequelae and are certainly more difficult to reverse. Clips made of plastic and metal are easy and safe to apply and damage only a very small piece of tube. Reversal of sterilisation is easiest after clips have been used because after the clip is excised the lumen of each piece of tube to be anastomosed is of equal diameter. Such operations may be performed successfully without the aid of microsurgical techniques (Noble, $\mathrm{AD}$, personal communication).

Women who have been sterilised seem to have a hysterectomy more often than those who have not, though the explanation is still not clear. ${ }^{7}$ The alleged association between sterilisation and menorrhagia is disputed: no large prospective trial has actually shown a measured increase in menstrual loss. Studying 10 patients for 12 months after sterilisation, Kasonde and Bonnar ${ }^{8}$ measured menstrual blood loss before and after the operation and found that some women had an increase and some a decrease-changes which cancelled each other out. Other retrospective studies have come to similar conclusions. Nevertheless, a diminution in bleeding is unlikely to trouble a woman, whereas an increased loss or severely abnormal rhythm may persuade her that she needs hysterectomy. 4910

One possibility is that sterilisation may impair the circulation between the uterus and ovary, and this might affect corpus luteum function. ${ }^{10}$ The results of recent studies support the possibility that plasma concentrations of progesterone may be reduced in patients who have been sterilised. ${ }^{11}$ Posttraumatic adhesions, an altered blood supply, and changed hormone balance provide a combination of objective changes that might easily disturb some patients. If such changes do indeed form the basis of the "poststerilisation syndrome" then the optimum method may be the one that causes the least trauma and the least change in the circulation.

Some surgeons advocate hysterectomy for sterilisation ${ }^{9} 1213$ -a logical choice in the presence of other symptoms attributable to pelvic disease. Since, however, modern sterilisation techniques have only modest immediate sequelae and recovery is so quick, few women are likely to prefer the major operation without very good reasons.

Most women are pleased with their decision to be sterilised, and have few regrets. The best results occur when the patient has made her own unhurried decision and at a time when she is not pregnant or recently pregnant. Women expecting that poor libido will be improved are usually disappointed. ${ }^{14}$ Pregnancy due to some operative failure is more likely if the patient was sterilised at the time of caesarean section or termination of pregnancy. ${ }^{13}$ Modern techniques are such that operation may reasonably be delayed until six weeks after childbirth or any convenient time thereafter. If this policy is followed, all cases will be elective and the risks of regret, failure, and surgical complications will all be reduced.

${ }^{1}$ Hughes G, Liston WA. Comparison between laparoscopic sterilisation and tubal ligation. $\mathrm{Br} \mathrm{Med} \mathcal{F} 1975$;ii i:637-9.

2 Letchworth AT, Kane L, Noble AD. Laparotomy or mini-laparotomy for female sterilisation. Obstet Gynecol 1980; in press.

${ }^{3}$ McCann MF. Laparoscopy versus mini-laparotomy. In: Schiara JJ, Zatuchi GI, Speidal JJ, eds. Risks, benefits and controversies in fertility control. New York: Harper and Row, 1978:68-80.

- Baggish MS, Lee WK, Miro SJ, Dacko L, Cogen G. Complications of laparoscopic sterilization. Comparison of 2 methods. Obstet Gynecol $1979 ; 54: 54-9$.

5 Lawson S, Cole RA, Templeton AA. The effect of laparoscopic sterilisation by diathermy or silastic bands on post-operative pain, menstrual symptoms and sexuality. Br $\mathcal{F}$ Obstet Gynaecol 1976;86:659-63.

- Pelland PC. Patient acceptance of laparoscopic tubal fulguration versus falope-ring banding. Obstet Gynecol 1977;50:106-8.

${ }^{7}$ Noble AD. Female sterilisation: long-term effects. In: Schiara JJ, Zatuchi GI, Speidel JJ, eds. Risks, benefits and controversies in fertility control. New York: Harper and Row, 1978:102-7.
8 Kasonde J, Bonnar J. Effect of sterilization on menstrual blood loss. Br F Obstet Gynaecol 1976;83:572-5.

Muldoon MJ. Gynaecological illness after sterilisation. Br Med $\mathcal{F} 1972$; i:85-5.

${ }^{10}$ Neil JR, Hammond GT, Noble AD, Rushton L, Letchworth AT. Late complications of sterilisation by laparoscopy and tubal ligation. A controlled study. Lancet 1975 ;ii:699-700.

11 Radwanska E, Berger GS, Hammond J. Luteal deficiency among women with normal menstrual cycles, requesting reversal of tubal sterilization. Obstet Gynecol 1979;54:789-92.

12 Scott JS. Gynaecological illness after sterilisation. Br Med F 1972;i:504.

13 Shepard MK. Female contraceptive sterilization. Obstet Gynecol Surv $1974 ; 29: 739-87$.

14 Sim M, Emens JM, Jordan JA. Psychiatric aspects of female sterilisation. Br Med f 1973;iii :220-2.

\section{Pyogenic liver abscess}

Pyogenic liver abscesses are rarely encountered in general hospital practice, but failure to recognise and treat the condition expeditiously almost always results in the patient's death. ${ }^{1-3}$ Its frequency has changed little over the past half century, though the type of patient, the ease of diagnosis, and, to some extent, the prognosis have altered. ${ }^{4-6}$ Whereas the disease used to affect younger people, often after appendicitis, and the patient usually had unambiguous symptoms and signs, ${ }^{78}$ today liver abscess occurs most often in patients in their 60 s with biliary disease or cancer. ${ }^{146} \mathrm{Up}$ to half the patients give no history of a predisposing illness. ${ }^{2}$ The symptoms and signs are mostly non-specific-malaise, anorexia, and fever-and often there is no clinical indication that the liver is the seat of the disease. ${ }^{6}$ All too often the diagnosis is made only at necropsy: in most large series about half the patients have died.1610

The most important factor in improving the prognosis is rapid diagnosis and an early start to treatment. ${ }^{1211}$ The morphology of the abscess is also important: patients with unilocular abscesses, usually confined to the right lobe of the liver, have a much better outlook (when adequately treated) than patients with multiple abscesses spread throughout the liver, ${ }^{14}$ in whom the outcome is almost always fatal whatever the treatment. The presence of jaundice usually denotes a multicentric abscess and is thus a poor prognostic sign. ${ }^{111}$ Older patients generally do worse than younger ones. ${ }^{14}$

Among the clinical features which might suggest the presence of liver abscess are tender enlargement of the liver together with fever in a jaundiced patient. This combination is present in not many more than half the cases, however, and even then a diagnosis of liver abscess is often overlooked in favour of more common upper abdominal inflammatory lesions such as pancreatitis and cholecystitis. ${ }^{2}$ More helpful clues include a raised serum concentration of alkaline phosphatase with a low concentration of albumin and a sedimentation rate of over $100 \mathrm{~mm}$ in one hour. Often the patient is anaemic. ${ }^{911}$ At the first suspicion of the possibility of an abscess the liver should be examined ultrasonically and by isotope scan; this will usually show a solitary abscess but not necessarily multicentric disease. ${ }^{2} 412$ Unfortunately, the false-negative rate seems as high as $25 \%$ with isotope scanning, so further investigation such as by computed tomography (or if this is not available hepatic arteriography) may be required. In the few cases where all localising investigations give negative results the surgeon must not be deterred from laparotomy if clinical suspicion remains high. 
Even at exploratory operation there may be difficulties. The affected area of the liver may be soft on palpation, but the liver may appear normal, when a large-bore needle must be passed into several areas to try to locate pus. In these circumstances preoperative real-time ultrasonography using a hand-held probe may be invaluable. ${ }^{13}$

When a unilocular abscess is found the cavity should be cleared, a large tube drain inserted, and treatment with appropriate antibiotics (such as gentamicin, metronidazole, and ampicillin) started immediately. Surgical drainage is undoubtedly inadequate alone. ${ }^{4}$ The drain should be left in place until there is sinographic evidence of obliteration of the cavity, and antibiotics should be continued for several weeks. ${ }^{11}$ With such a regimen $90 \%$ of patients with solitary abscesses will survive-an improvement on results from the preantibiotic era. ${ }^{4}$ In patients with multicentric abscesses the prognosis remains poor owing to the difficulty of obtaining satisfactory surgical drainage; fewer than a fifth of such patients will survive. ${ }^{134}$

${ }^{1}$ Heymann AD. Clinical aspects of grave pyogenic abscesses of the liver. Surg Gynecol Obstet 1979;149:209-13.

2 Silver S, Weinstein A, Cooperman A. Changes in the pathogenesis and detection of intrahepatic abscess. Am $\mathcal{f}$ Surg 1979;137:608-10.

3 Brodine WN, Schwartz SI. Pyogenic hepatic abscess. NY State 7 Med 1973;73:1657-61.

- Pitt HA, Zuidema GD. Factors influencing mortality in the treatment of pyogenic hepatic abscess. Surg Gynecol Obstet 1975;140:228-34.

5 Sherman JD, Robbins SL. Changing trends in the casuistics of hepatic abscess. $A m \mathcal{F}$ Med 1960;28:943-50.

- Rubin RH, Swartz MN, Malt R. Hepatic abscess: changes in clinical, bacteriologic and therapeutic aspects. Am $\mathcal{F}$ Med 1974;57:601-10.

7 Ochsner A, DeBakey M, Murray S. Pyogenic abscess of the liver. $\mathrm{Am} \mathcal{F}$ Surg 1938;11:292-319.

8 Palmer ED. The changing manifestations of pyogenic liver abscess. fAMA 1975;231:192.

- Young AE. The clinical presentation of pyogenic liver abscess. Br $\mathcal{F}$ Surg $1976 ; 63: 216-9$.

10 Schraibman IG. Non-parasitic liver abscess. Br $\mathcal{f}$ Surg 1974;61:709-12.

11 Butler TJ, McCarthy CF. Pyogenic liver abscess. Gut 1969;10:389-99.

12 Lawson TL. Hepatic abscess-ultrasound as an aid to diagnosis. Am $\mathcal{f}$ Dig Dis 1977;22:33-7.

${ }^{13}$ Lytton B, Cook J. Intraoperative ultrasound. In: Resnick MI, Saunders RC, eds. Ultrasound in urology. Baltimore: Williams and Wilkins, 1979:340-51.

\section{Is malignant diverticulitis a true bill?}

A recent report ${ }^{1}$ from California described 17 patients with a new clinical entity labelled "malignant" diverticulitis and characterised by extensive inflammation of the sigmoid and rectosigmoid, affecting all pelvic structures and often extending below the peritoneal reflection. Both obstruction and perforation were common, and many of the patients developed fistulas into adjacent viscera and on to the skin. Despite treatment with antibiotics, diversion of the faecal stream by colostomy, and other measures, a high proportion had postoperative complications and three died. The term "malignant" was applied because the diverticulitis behaved differently from ordinary diverticulitis in its progression and severity; there was no implication of neoplastic change. Nine out of 12 patients who had a colonic resection developed anastomotic leaks with ensuing sepsis and fistulas-a frightening complication rate by any standards, and one suggesting that there must have been some underlying cause for such alarming results.
Diverticula are never present in the rectum, ${ }^{2}$ and diverticulitis rarely affects more than the sigmoid colon. An inflammatory lesion of unusual extent, as in this so-called "malignant" diverticulitis, suggests some additional disease process. That was the case in the series described by Schmidt and his colleagues ${ }^{3}$ from St Mark's Hospital, London, of 26 patients with associated diverticulitis and Crohn's disease. Nine of their patients with a preoperative diagnosis of diverticulitis were subsequently shown to have coincidental Crohn's disease, and six developed postoperative complications of anastomotic leak, fistula formation, and sepsisalmost identical with those of "malignant" diverticulitis. In comparison, of 50 patients operated on at St Mark's Hospital for diverticulitis alone, only one developed a postoperative complication, an anal fistula.

In both the London and the California series the inflammation was intramural, severe, and extensive-a pattern more characteristic of Crohn's disease than diverticulitis alone, where the inflammation is mainly pericolic and extramural. Morgenstern et $a l^{1}$ admit the clinical similarity of "malignant" diverticulitis and granulomatous colitis but the pathological findings are, they say, consistent with severe diverticulitis. Nevertheless, the radiographic evidence of long intramural sinus tracts and the development of fistulas in 12 of their 17 patients are further evidence in favour of Crohn's disease rather than diverticulitis alone.

No mention is made in the report of the sigmoidoscopic findings, which could have been an important clue in the diagnosis of possible associated Crohn's disease. Anal lesions, mucosal granularity, pus, and ulceration suggest inflammatory bowel disease. A rectal biopsy is mandatory in patients with combined disease as there is a high incidence of abnormality. Morgenstern et al emphasise the absence of mucosal changes in their patients, though naked eye appearances alone do not entirely rule out the diagnosis of Crohn's disease.

Nor is anything said of the specific antibiotics used or of the culture of organisms found in the bowel lumen or in abscesses. Gram-negative anaerobes may have been responsible for the extensive inflammatory process that occurred in these 17 patients.

Granulomatous disease is known to coexist with diverticulitis in some patients and especially in the sigmoid colon in elderly women; and granulomatous colitis may masquerade as acute diverticulitis. ${ }^{4}$ Generally the distinction presents little diagnostic difficulty; but some patients may pose problems, especially after surgery for diverticulitis, when postoperative complications may be due to failure to recognise granulomatous disease. These patients are more likely to benefit from treatment with corticosteroids than from radical surgery.

The introduction of a new concept of "malignant" diverticulitis seems premature on the evidence so far available. When severe and progressive diverticulitis occurs in elderly patients with a high incidence of postoperative complications, extensive intramural inflammation, and the long sinus tracts, the doctor should assume that Crohn's disease is also present until the contrary is clearly proved.

1 Morgenstern L, Weiner R, Michel SL. "Malignant" diverticulitis a clinical entity. Arch Surg 1979;114:1112-6.

${ }^{2}$ Morson BC, Dawson IPM. Gastrointestinal pathology. Oxford: Blackwell, $1974: 499$.

s Schmidt GT, Lennard-Jones JE, Morson BC, Young AC. Crohn's disease of the colon and its distinction from diverticulitis. Gut 1968; 9:7-16.

4 Hoffman WA, Rosenberg MA. Granulomatous colitis in the elderly. Am $\mathcal{F}$ Gastroenterol 1972;58:508-18. 